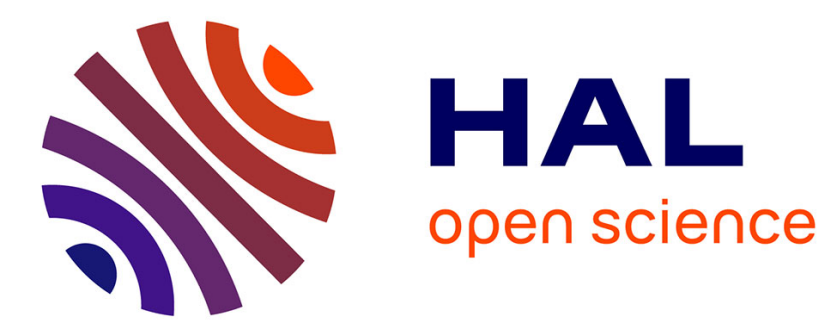

\title{
Opportunistic Secondary Spectrum Sharing Protocols for Primary implementing an IR type Hybrid-ARQ Protocol
}

Romain Tajan, Charly Poulliat, Inbar Fijalkow

\section{- To cite this version:}

Romain Tajan, Charly Poulliat, Inbar Fijalkow. Opportunistic Secondary Spectrum Sharing Protocols for Primary implementing an IR type Hybrid-ARQ Protocol. IEEE International Conference on Acoustics, Speech, and Signal Processing (ICASSP) 2012, Mar 2012, Kyoto, Japan. 4 p. hal-00671053

\section{HAL Id: hal-00671053 \\ https://hal.science/hal-00671053}

Submitted on 16 May 2012

HAL is a multi-disciplinary open access archive for the deposit and dissemination of scientific research documents, whether they are published or not. The documents may come from teaching and research institutions in France or abroad, or from public or private research centers.
L'archive ouverte pluridisciplinaire HAL, est destinée au dépôt et à la diffusion de documents scientifiques de niveau recherche, publiés ou non, émanant des établissements d'enseignement et de recherche français ou étrangers, des laboratoires publics ou privés. 


\title{
OPPORTUNISTIC SECONDARY SPECTRUM SHARING PROTOCOLS FOR PRIMARY IMPLEMENTING AN IR TYPE HYBRID-ARQ PROTOCOL
}

\author{
Romain Tajan*, Charly Poulliat ${ }^{\dagger}$, Inbar Fijalkow* \\ * ETIS / ENSEA - University of Cergy-Pontoise - CNRS, France \\ ${ }^{\dagger}$ IRIT/ INP-ENSEEIHT, University of Toulouse, France \\ \{romain.tajan, inbar.fijalkow\}@ensea.fr, charly.poulliat@enseeiht.fr
}

\begin{abstract}
In this paper, we propose, analyse and compare three different methods for opportunistic spectrum sharing access when the primary users implements an Incremental Redundancy (IR) type Hybrid Automatic ReQuest (H-ARQ) protocol. The first method consists in allowing the secondary user to communicate only during the first primary transmission round of the IR H-ARQ protocol. In this scenario, if the the secondary receiver fails to decode its message after the first round, it realizes a successive interference cancellation in the subsequent primary HARQ rounds by listening to the primary user. The second method consists in realizing a perfect interference cancellation at the secondary receiver with causal channel state information. In this method, the secondary user communicates only when the secondary receiver succeeds in decoding the primary message. To improve throughput performance at the secondary, the secondary pair is also considering the use of an IR-HARQ protocol. In a third method, the secondary user communicates following the same rule as in the proposed second method, but implementing an Adaptive Modulation and Coding scheme instead of HARQ. In particular, we show that this last protocol with a small number of interfered slots allows to limit the loss in the primary throughput needed for the secondary user to transmit.
\end{abstract}

Index Terms - Spectrum Sharing, Cognitive Radio, HARQ, AMC, Throughput

\section{INTRODUCTION}

In Cognitive Radio, two access strategies are generally considered in order to improve the spectrum access efficiency. The first strategy, called Opportunistic Spectrum Access, consists in detecting spectrum white spaces and communicating over those available spaces. For the second method instead, called Opportunistic Spectrum Sharing, both primary and secondary users share the same spectrum at the same time. The model for this channel is often referred to as the cognitive channel and has been introduced in [1]. Throughout this paper, we consider the second strategy as a framework for our study.

In the spectrum sharing context, different secondary protocols have been proposed. The developed protocols mainly differs from the different access protocols and link adaptation strategies considered for the primary users (ie. rate, power or combined adaptation strategies). Apart from power allocation only strategies as done in $[2,3]$, several contributions have considered rate adaptation with Adaptive Modulation and Coding (AMC) scheme at the secondary (see for example $[4,5]$ and references therein), where the ergodic capacity is often used as a measure of performance. In particular

For this work R. Tajan was supported by DGA and CNRS in [5], the primary users implement an AMC scheme with a training period and use that period in order to adapt to the channel variations. The secondary users communicate over this training period in order to enforce primary users to adapt their rate and power when facing secondary interferences. Authors have shown that using this active learning can improve the secondary performances under constraint of primary losses. Another strategy to perform rate adaptation is the use of Hybrid Automatic ReQuest (HARQ) mechanisms to adapt to the channel variations and to cope with other types of interferences. This strategy has been first considered in $[6,7]$ for a cognitive channel ( [8]). In these works, the primary users implement an HARQ with only two HARQ transmission rounds. In both cases, the channel is assumed quasi-static fading Rayleigh channel where the channel gains remain constant over the whole duration of the protocol. In [7], the authors consider that more than one primary packet can be transmitted over one time coherence and propose four different secondary protocols depending on the primary state over the two transmissions. The performances of the system are evaluated in terms of goodput. In [6], only one primary packet (possibly two transmissions) can be sent during one coherence time. In both cases, the secondary users intend to exploit the opportunity to communicate during the retransmission HARQ rounds. This is achieved by listening to the first HARQ round of the primary, and then, depending on the secondary Channel State Information (CSI), the secondary pair intends to realize perfect interference cancellation, dirty-paper coding [8] or power control. The performances are given in terms of outage probability and expected throughput. Note that in $[6,7]$, no rate (eg. HARQ/AMC) and/or power adaptation is considered for the secondary users when accessing the cognitive channel.

In this article, we consider rate adaptation strategies for the secondary users when primary users implement a slotted Incremental Redundancy type HARQ (IR-HARQ) with an arbitrary number of retransmission rounds and with rounds of different sizes. Contrary to [6,7], we consider a slotted block-fading channel where the Rayleigh coefficients change independently at each slot. In this context, we intend to compare three different protocols for the secondary users. The first protocol considers that the secondary users always communicate on the first primary HARQ round only and then try to realize successive interference cancellation by listening to the subsequent primary HARQ rounds if decoding of the secondary message failed. We refer to this policy as HARQ with successive interference cancellation. The second proposed policy is referred to as $H A R Q$ with perfect interference cancellation: the secondary system communicates only when it can realize perfect interference cancellation, ie. the secondary is listening to the primary user during its first HARQ round and then tries to decode. We further assume that the secondary pair uses an IR-HARQ protocol. The third proposed 


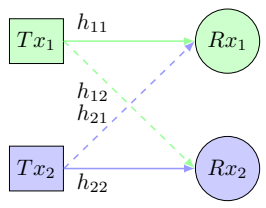

Fig. 1. Interference Channel

policy is similar to the second one and is referred to as AMC with perfect interference cancellation. For this policy, the secondary user also communicates only when it can realize perfect interference cancellation, but AMC is used instead of HARQ to adapt to the channel variation. For all these protocols, we perform a performance analysis in term of long-term throughput(cf. $[9,10])$, since ergodic capacity cannot be used in an HARQ context.

This paper is organized as follows. In Section 2, we present the channel model and the different protocols considered for both primary and secondary users. In Section 3, we give the performance analysis of the studied systems in term of long-term throughput. In Section 4, some simulation results are given. Finally Section 5 concludes this paper.

\section{CHANNEL AND SYSTEM MODEL}

For the ease of presentation, we consider in this paper a simple network composed by a primary pair transmitter/receiver $\left(T x_{1}, R x_{1}\right)$ and a cognitive pair transmitter/receiver $\left(T x_{2}, R x_{2}\right)$ as presented in Figure 1. We consider that each channel link is a block-fading Additive White Gaussian Noise (AWGN) channel. The transmissions over these channels are slotted and each slot is assume to last $L$ channel use (cu). The fading coefficients of the channel links remain constant over all the duration of a slot. Therefore, the model for the time slot $m$ can be expressed by

$$
\begin{aligned}
& \mathbf{y}_{1}(m)=h_{11}(m) \mathbf{x}_{1}(m)+h_{21}(m) \mathbf{x}_{2}(m)+\mathbf{z}_{1}(m) \\
& \mathbf{y}_{2}(m)=h_{12}(m) \mathbf{x}_{1}(m)+h_{22}(m) \mathbf{x}_{2}(m)+\mathbf{z}_{2}(m)
\end{aligned}
$$

where $\mathbf{x}_{1}(m), \mathbf{x}_{2}(m) \in \mathbb{C}^{L}$ are the messages issues from Gaussian codebooks transmitted by $T x_{1}$ and $T x_{2}$ respectively. $\mathbf{y}_{1}(m)$, $\mathbf{y}_{2}(m) \in \mathbb{C}^{L}$ are the messages received at $R x_{1}$ and $R x_{2}$ respectively. The channel coefficients $h_{i j}(m)$ are independent complex random Gaussian variables such as $\alpha_{i j}(m)=\left|h_{i j}(m)\right|^{2}$ are exponential random variables with mean $\overline{\alpha_{i j}}$. The Signal to Noise Ratio (SNR) for the link $i j$ during the time slot $m$ is denoted as $\gamma_{i j}(m) \triangleq P_{i} \alpha_{i j}(m)$. The Signal to interference plus Noise Ratio (SINR) at the receiver $i$ is defined as $\beta_{i}(m) \triangleq \frac{\gamma_{i i}(m)}{1+\gamma_{j i}(m)}$.

If the secondary succeeds in decoding the primary message, we consider that the resulting interference cancellation is perfect leading to $\beta_{2}(m)=\gamma_{22}(m)$.

\subsection{Primary Protocol}

The primary pair implements a slotted Incremental Redundancy Hybrid-ARQ protocol (IR-HARQ) with infinite buffer at the emitter (fully-loaded queues) using one bit feedback for acknowledgement in order to adapt its rate to the channel variations $[9,10]$. This protocol can be described as follows: $T x_{1}$ encodes an information packet of size $b_{1}$ bits into a $N$ code blocks $\mathbf{x}_{1}, \mathbf{x}_{2}, \ldots, \mathbf{x}_{N}$ where $\mathbf{x}_{1}$ has a duration of $B_{1}$ slots and all other $\mathbf{x}_{i}$ have a duration of one slot. Let the total number of slots of the policy be denoted by $N_{1}=B_{1}+N-1$. Let also introduce the rate of the first slot: $r_{1}=\frac{b_{1}}{L}$. The primary user $T x_{1}$ sends $\mathbf{x}_{1}$ through the channel to
$R x_{1}$ who then tries to decode. If the decoding at $R x_{1}$ is successful, $R x_{1}$ sends an ACKnowledgment (ACK) feedback bit to $T x_{1}$ who generates a new information packet. This happens (cf. [10]) if $\sum_{s=1}^{B_{1}} C\left(\gamma_{11}(s)\right) \geq r_{1}$ where $C\left(\gamma_{11}(s)\right)=\log _{2}\left(1+\gamma_{11}(s)\right)$ is the instantaneous mutual information of the channel on slot $s$. If the decoding at $R x_{1}$ fails $\left(\sum_{s=1}^{B_{1}} C\left(\gamma_{11}(s)\right)<r_{1}\right), R x_{1}$ sends a Negative ACKnowledgment (NACK) feedback bit to $T x_{1}$ who sends the next code block in the IR code policy. The HARQ process continues until either $R x_{1}$ successfully decodes the information packet or the $N$ code blocks are sent.

\subsection{Secondary Protocols}

In this subsection, we describe three protocols for the secondary users. The main idea of these three protocols is that the secondary users intend to benefit from interference cancellation of the primary user. For all these protocols, the considered feedback channels are assumed instantaneous and perfect (error-free feedbacks).

\section{IR-HARQ with successive interference cancellation}

This protocol can be seen as a generalization of what is proposed in [6] as a perspective. Note however that the channel considered here is the slotted block-fading channel compared to the quasi-static block fading channel as considered in [6]. The proposed protocol can be divided into two steps: (1) during the first primary HARQ round (ie. the first $B_{1}$ slots), $R x_{2}$ considers the message sent by $T x_{1}$ as noise to attempt, at each new slot, to decode its own message using an IR-HARQ protocol on a slot basis. To do so, $T x_{2}$ encodes $b_{2}$ information bits into $N_{2}$ code blocks of length $L$. The rate of the first slot is denoted by $r_{2}=\frac{b_{2}}{L}$. (2) If decoding has failed after sending its $N_{2}$ blocks (with $N_{2} \leq B_{1}$ ), the secondary transmitter keeps quiet and the secondary receiver listens for the primary messages. If the secondary receiver succeeds in decoding the primary information at a new primary HARQ round, it uses this information to realize perfect interference cancellation to decode its own message.

\section{IR HARQ with Perfect Interference Cancellation}

This protocol can also be divided into two parts: (1) $R x_{2}$ listens to the primary link. If $R x_{2}$ succeeds in decoding the primary information before $R x_{1}$, it sends a Clear To Send (CTS) message to $T x_{2}$ through the feedback channel, (2) then, at the reception of the CTS message, $T x_{2}$ and $R x_{2}$ implement an IR-HARQ protocol with $N_{2}$ possible transmissions. Given that this protocol starts only if $R x_{2}$ possesses the primary information, it can perform perfect interference cancellation.

\section{AMC with Perfect Interference Cancellation}

The third protocol is similar to the second one but implements an AMC scheme instead of an HARQ protocol during the second phase of the protocol. We consider that $R x_{2}$ perfectly knows the $h_{22}(m)$ amplitude and phase for the slots on which it can communicate. Note that this CSI is not required for the two other protocols.

\section{PERFORMANCE ANALYSIS}

For both primary and secondary systems, the performance is given in term of throughput which is here define as the number of correctly received bits per unit of time. The computation of the throughput using renewal reward theory was firstly introduced by [11] and is used also in $[9,10]$. 


\subsection{Performance of the Primary System with Silent Secondary}

The computation of the performance of the performance of the primary system alone is similar to the computation given in [10]. Let introduce the recurrent event at $R x_{1}$ as the transmitter $R x_{1}$ stops transmitting the current codeword. For each recurrent event, we attach a random reward $\mathcal{R}_{1}$ wich is equal to $b_{1}$ bits if the packet is successfully decoded at the receiver and is equal to 0 bit if not. The corresponding long-term throughput can be defined as

$$
\eta_{1}=\lim _{t \rightarrow \infty} \frac{b_{1}(t)}{t}=\frac{\mathbb{E}\left(\mathcal{R}_{1}\right)}{\mathbb{E}\left(\mathcal{T}_{1}\right)}
$$

where $b_{1}(t)$ represent the total number of information bits received at $R x_{1}$ between time 0 and time $t$ and where the right-hand part of this equation is due to the renewal theory with $\mathcal{T}_{1}$ being the random time between two successive recurrent events. Let $p_{1}(m)$ be probability that $R x_{1}$ cannot decode after $m$ slots, $p_{1}(m)$ given by

$$
p_{1}(m)=\mathbb{P}\left(\sum_{j=1}^{m} C\left(\gamma_{11}(j)\right)<r_{1}\right)
$$

Finally, the throughput of the primary system is given by

$$
\eta_{1}=r_{1} \frac{1-p_{1}\left(N_{1}\right)}{B_{1}+\sum_{m=1}^{N_{1}-1} p_{1}(m)}
$$

\subsection{Performance of the Primary and Secondary Systems}

\section{IR-HARQ with Successive Interference Cancellation}

In this section, the secondary user implements the protocol described in 2.2. In order to evaluate the performances of the primary and secondary systems, let us introduce the following random variables: $\mathcal{K}$ which is counting the time that $R x_{2}$ needs in order to correctly decode its current packet if we suppose $N_{2}=\infty$ and $\mathcal{I}$ which is counting the number of slots on which the secondary user is communicating. The probability density of $\mathcal{K}$ is given by

$$
\mathbb{P}(\mathcal{K}=k)=\mathbb{P}\left(\sum_{m=1}^{k} C\left(\beta_{2}(m)\right) \geq r_{2}, \sum_{m=1}^{k-1} C\left(\beta_{2}(m)\right)<r_{2}\right) .
$$

The probability density of $\mathcal{I}$ is obtained considering that if $\mathcal{K} \leq$ $N_{2}$ then $\mathcal{I}=\mathcal{K}$ and if $\mathcal{K}>N_{2}$ then $\mathcal{I}=N_{2}$.

$$
\mathbb{P}(\mathcal{I}=i)=\left\{\begin{array}{l}
\mathbb{P}(\mathcal{K}=i) \text { if } i<N_{2}, \\
\mathbb{P}\left(\mathcal{K} \geq N_{2}\right) \text { if } i=N_{2},
\end{array}\right.
$$

Using these two quantities, the throughput of the primary system can then be written as

$$
\eta_{1}=\frac{\mathbb{E}_{\mathcal{I}}\left(\mathbb{E}\left(\mathcal{R}_{1} \mid \mathcal{I}\right)\right)}{\mathbb{E}_{\mathcal{I}}\left(\mathbb{E}\left(\mathcal{T}_{1} \mid \mathcal{I}\right)\right)}
$$

which modifies equation (4) as follows

$$
\eta_{1}=r_{1} \frac{1-\mathbb{E}_{\mathcal{I}}\left(p_{1}\left(N_{1} \mid \mathcal{I}\right)\right)}{B_{1}+\sum_{m=1}^{N_{1}-1} \mathbb{E}_{\mathcal{I}}\left(p_{1}(m \mid \mathcal{I})\right)},
$$

where

$$
\mathbb{E}_{\mathcal{I}}\left(p_{1}(m \mid \mathcal{I})\right)=\sum_{i=1}^{N_{2}} f(m, i) \mathbb{P}(\mathcal{I}=i)
$$

with $f(m, i)=\mathbb{P}\left(\sum_{s=1}^{i} C\left(\beta_{1}(s)\right)+\sum_{s=i+1}^{m} C\left(\gamma_{11}(s)\right)<r_{1}\right)$.
The throughput of the secondary user can be defined in a similar way to the primary user as follows

$$
\eta_{2}=\lim _{t \rightarrow \infty} \frac{b_{2}(t)}{t}=\frac{\mathbb{E}\left(\mathcal{R}_{2}\right)}{\mathbb{E}\left(\mathcal{T}_{2}\right)}
$$

In this paper, we consider that, for each transmission of a new packet by the primary user, the secondary user also starts a new transmission which implies that $\mathbb{E}\left(\mathcal{T}_{2}\right)=\mathbb{E}\left(\mathcal{T}_{1}\right)$. The proof of this assertion is not given in this paper but remains true for the three secondary protocols. The computation of $\mathbb{E}\left(\mathcal{R}_{2}\right)$ in this case can be simplified by noticing that $\left\{\mathcal{R}_{2}=0\right\}$ is equivalent to $\left\{\sum_{s=1}^{N_{2}} C\left(\gamma_{22}(s)\right)<r_{2}\right\}$. The secondary throughput is finally given by

$$
\eta_{2}=r_{2} \frac{\mathbb{P}\left(\sum_{s=1}^{N_{2}} C\left(\gamma_{22}(s)\right)<r_{2}\right)}{B_{1}+\sum_{m=1}^{N_{1}-1} \mathbb{E}_{\mathcal{I}}\left(p_{1}(m \mid \mathcal{I})\right)}
$$

\section{HARQ with Perfect Interference Cancellation}

For this analysis, we keep the random variable $\mathcal{K}$ and we add the random variable $\mathcal{J}$ which represents the number of primary slots that $R x_{2}$ needs in order to correctly decode the information sent by $T x_{1}$ supposing $N_{1}=\infty$. The probability density of $\mathcal{J}$ is

$$
\mathbb{P}(\mathcal{J}=j)=\mathbb{P}\left(\sum_{s=1}^{j} C\left(\gamma_{12}(s)\right) \geq r_{1}, \sum_{s=1}^{j-1} C\left(\gamma_{12}(s)\right)<r_{1}\right)
$$

Using $\mathcal{K}$ and $\mathcal{J}$, the primary throughput is given by

$\eta_{1}=\frac{\mathbb{E}_{\mathcal{J}, \mathcal{K}}\left(\mathbb{E}\left(\mathcal{R}_{1} \mid \mathcal{J}, \mathcal{K}\right)\right)}{\mathbb{E}_{\mathcal{J}, \mathcal{K}}\left(\mathbb{E}\left(\mathcal{T}_{1} \mid \mathcal{J}, \mathcal{K}\right)\right)}=r_{1} \frac{1-\mathbb{E}_{\mathcal{J}, \mathcal{K}}\left(p_{1}\left(N_{1} \mid \mathcal{J}, \mathcal{K}\right)\right)}{B_{1}+\sum_{m=1}^{N_{1}-1} \mathbb{E}_{\mathcal{J}, \mathcal{K}}\left(p_{1}(m \mid \mathcal{J}, \mathcal{K})\right)}$,

where

$\mathbb{E}_{\mathcal{J}, \mathcal{K}}\left(p_{1}(m \mid \mathcal{J}, \mathcal{K})\right)=\sum_{j=B_{1}}^{N_{1}} \sum_{k=1}^{N_{2}} g(m, j, k) \mathbb{P}(\mathcal{J}=j) \mathbb{P}(\mathcal{K}=k)$,

with $g(m, j, k)=\left\{\begin{array}{l}f(m, 0) \text { if } m \leq j, \\ f(m, k) \text { if } m \geq j+k+1 \text { and } k<N_{2}, \\ f\left(m, N_{2}\right) \text { if } m \geq j+N_{2}+1 \text { and } k \geq N_{2}, \\ f(m, m-j) \text { if } j \leq m \leq j+k,\end{array}\right.$

The secondary throughput is still given by equation (10) where we also have $\mathbb{E}\left(\mathcal{T}_{2}\right)=\mathbb{E}\left(\mathcal{T}_{1}\right)$ and $\mathbb{E}\left(\mathcal{R}_{2}\right)=\mathbb{E}_{\mathcal{J}, \mathcal{K}}\left(\mathbb{E}\left(\mathcal{R}_{2} \mid \mathcal{J}, \mathcal{K}\right)\right)$ and $\eta_{2}$ is given by

$$
\eta_{2}=r_{2} \frac{1-\sum_{k=1}^{N_{2}} \sum_{j=B_{1}}^{N_{1}-k} f(j+k, k) \mathbb{P}(\mathcal{J}=j) \mathbb{P}(\mathcal{K}=k)}{B_{1}+\sum_{m=1}^{N_{1}-1} \mathbb{E}_{\mathcal{J}, \mathcal{K}}\left(p_{1}(m \mid \mathcal{J}, \mathcal{K})\right)}
$$

\section{AMC with Perfect Interference Cancellation}

Using the same method as the two precedent analysis, we have

$$
\eta_{1}=\frac{\mathbb{E}_{\mathcal{J}}\left(\mathbb{E}\left(\mathcal{R}_{1} \mid \mathcal{J}\right)\right)}{\mathbb{E}_{\mathcal{J}}\left(\mathbb{E}\left(\mathcal{T}_{1} \mid \mathcal{J}\right)\right)}=r_{1} \frac{1-\mathbb{E}_{\mathcal{J}}\left(p_{1}\left(N_{1} \mid \mathcal{J}\right)\right)}{B_{1}+\sum_{m=1}^{N_{1}-1} \mathbb{E}_{\mathcal{J}}\left(p_{1}(m \mid \mathcal{J})\right)},
$$

where

$$
p_{1}(m \mid \mathcal{J}=j)=\left\{\begin{array}{l}
f(m, 0) \text { if } m \leq j \\
f(m, m-j) \text { if } j \leq m \leq N_{2}, \\
f\left(m, N_{2}\right) \text { if } m \geq N_{2}
\end{array}\right.
$$

In order to compute the secondary throughput, we need to carefully specify $\mathcal{R}_{2}$. In this case, we have $\mathcal{R}_{2}=L \sum_{s=1}^{\mathcal{N}} C\left(\gamma_{22}(s)\right)$, 
where $\mathcal{N}$ is a random variable representing the number of slots on which $T x_{2}$ has sent packets during one primary transmission. We then redefine $N_{2}$ as the maximum slots on which the secondary can communicate, thus $\mathcal{N} \in\left[1, N_{2}\right]$. Given the definition of $\mathcal{N}$ and the protocol, $\mathcal{N}$ depends on the length of the primary protocol (which depends on $h_{11}$ and $h_{21}$ ) and on the time that $R x_{2}$ decode the message of $T x_{1}$ (which depends only on $h_{12}$ ), thus $\mathcal{N}$ is independent of $h_{22}$. Because the $\gamma_{22}(s)$ are i.i.d, we can rewrite $\mathbb{E}\left(\mathcal{R}_{2}\right)$ as follows

$$
\mathbb{E}\left(\mathcal{R}_{2}\right)=\mathbb{E}_{\mathcal{N}}\left(\mathbb{E}\left(\mathcal{R}_{2} \mid \mathcal{N}\right)\right)=L \mathbb{E}(\mathcal{N}) \mathbb{E}\left(C\left(\gamma_{22}\right)\right),
$$

where $\mathbb{E}(\mathcal{N})$ is given by

$$
\mathbb{E}(\mathcal{N})=\sum_{n=1}^{N_{2}} n \sum_{j=B_{1}}^{N_{1}-n} f(j+n-1, n-1) \mathbb{P}(\mathcal{J}=j)
$$

and $\mathbb{E}\left(C\left(\gamma_{22}\right)\right)$ is computed using the pdf of $\gamma_{22}$ which is exponential. For this protocol, it can also be shown that $\mathbb{E}\left(\mathcal{T}_{2}\right)=\mathbb{E}\left(\mathcal{T}_{1}\right)$ which give the following result

$\eta_{2}=\frac{\sum_{n=1}^{N_{2}} n \sum_{j=B_{1}}^{N_{1}-n} f(j+n-1, n-1) \mathbb{P}(\mathcal{J}=j) \mathbb{E}\left(C\left(\gamma_{22}\right)\right)}{B_{1}+\sum_{m=1}^{N_{1}-1} \mathbb{E}_{\mathcal{J}}\left(p_{1}(m \mid \mathcal{J})\right)}$.

\section{RESULTS}

In this section, we compare the three proposed secondary strategies. The primary users communicate with a power of $10 \mathrm{dBW}$ and implement an IR-HARQ with $B_{1}=4$ slots, $N_{1}=8$ slots, $b_{1}=1024$ bits, $L=215 \mathrm{cu}$ which leads $r_{1}$ to be $r_{1} \approx 4.76 \mathrm{bpcu}$. This values of $L$ and $r_{1}$ have been chosen in order to maximise the throughput of the primary user when he is alone.

Five different secondary strategies have been tested for different secondary powers $P_{2}$ going from $-20 \mathrm{dBW}$ to $10 \mathrm{dBW}$ :

$s_{1}$ HARQ with successive interference cancellation with $N_{2}=3$,

$s_{2}$ HARQ with perfect interference cancellation with $N_{2}=3$,

$s_{3}$ AMC with perfect interference cancellation with $N_{2}=\infty$,

$s_{4}$ AMC with perfect interference cancellation with $N_{2}=3$ and

$s_{5}$ AMC with perfect interference cancellation with $N_{2}=1$.

For the two strategies $s_{1}$ and $s_{2}$ and for each power, we have considered secondary rates going from 0 bpcu to $20 \mathrm{bpcu}$ and we have kept the one maximizing the secondary throughput. The throughputs for both primary and secondary users for the different strategies are presented in Figure 2.

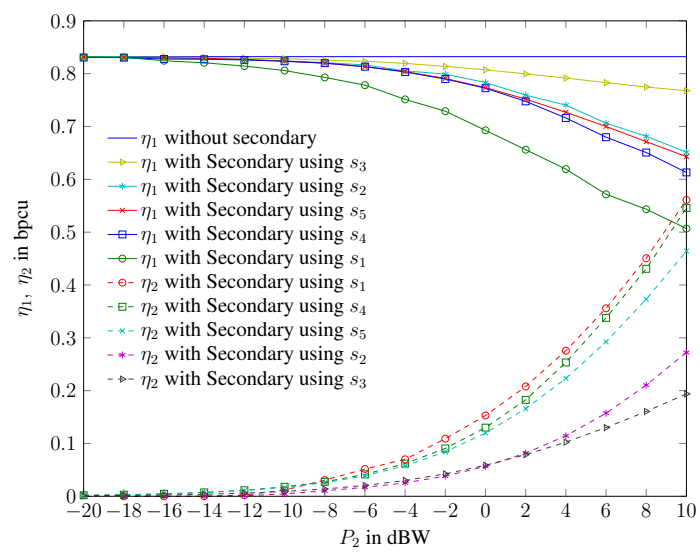

Fig. 2. Achievable throughputs for the proposed protocols versus the secondary transmit power
On these curves, one can remark that $N_{2}$ allows to trade off between the fairness with the primary $\left(N_{2}=1\right)$ and the secondary throughput $\left(N_{2}=\infty\right)$ for AMC based protocols. In fact, it can be easily proven that $\eta_{1}\left(s_{1}\right) \geq \eta_{1}\left(s_{2}\right) \geq \eta_{1}\left(s_{5}\right)$ just by noticing that the expected number of interfered blocks will be higher for $s_{1}$ than for $s_{2}$ which will be higher for $s_{2}$ than for $s_{3}$. This is due to the fact that HARQ protocols require more slots in order to decode their data. Finally we can observe that the AMC protocol with a small number of interfered slots allows a small loss in the primary throughput.

\section{CONCLUSION}

In this paper, we analyse the throughput of three different secondary strategies when the primary users implement an IR-HARQ protocol. If the primary system implements an HARQ protocol with a multiple bits feedback, a power control can be used on top of one of the proposed strategies to control the primary loss in throughput. This strategy will be considered in a futur work.

\section{REFERENCES}

[1] P. Mitran N. Devroye and V. Tarokh, "Achievable rates in cognitive radio channels," IEEE Tr. on IT, vol. 52, no. 2, pp. 18131827, May 2006.

[2] A. Bagayoko, I. Fijalkow, and P. Tortelier, "Power control of spectrum-sharing in fading environment with partial channel state information," IEEE Tr. on Signal Proc, vol. 59, no. 5, pp. $2244-2256$, may 2011.

[3] X. Kang, R. Zhang, Y-C. Liang, and H.K. Garg, "Optimal power allocation strategies for fading cognitive radio channels with primary user outage constraint," IEEE Journal on Selected Areas in Comm., vol. 29, no. 2, pp. 374 -383, feb. 2011.

[4] V. Asghari and S. Aissa, "Adaptive rate and power transmission in spectrum-sharing systems," IEEE Tr. on Wireless Comm., vol. 9, no. 10, pp. $3272-3280$, october 2010.

[5] R. Zhang, "On active learning and supervised transmission of spectrum sharing based cognitive radios by exploiting hidden primary radio feedback," IEEE Tr. on Comm., vol. 58, no. 10, pp. $2960-2970$, october 2010.

[6] R. A. Tannious and A. Nosratinia, "Cognitive radio protocols based on exploiting Hybrid-ARQ retransmissions," IEEE Tr. on Wireless Comm., vol. 9, no. 9, pp. 2833-2841, Sept. 2010.

[7] J. C. F. Li, W. Zhang, A. Nosratinia, and J. Yuan, "Opportunistic spectrum sharing based on exploiting arq retransmission in cognitive radio networks.," in GLOBECOM'10, 2010, pp. 1-5.

[8] A. Jovicic and P. Viswanath, "Cognitive radio: An information-theoretic perspective," IEEE Tr. on IT, vol. 55, no. 9, pp. 3945-3958, Sept. 2009.

[9] G. Caire and D. Tuninetti, "The throughput of Hybrid-ARQ protocols for the gaussian collision channel," IEEE Tr. on IT, vol. 47, no. 5, pp. 1971-1988, July 2001.

[10] S. Sesia, G. Caire, and G. Vivier, "Incremental redundancy Hybrid-ARQ schemes based on low-density paritycheck codes," IEEE Tr. on Comm., vol. 52, no. 8, pp. 13111321, Aug. 2004.

[11] M. Zorzi and R. R. Rao, "On the use of renewal theory in the analysis of ARQ protocols," IEEE Tr. on Comm., vol. 44, no. 9, pp. 1077-1081, Sept. 1996. 